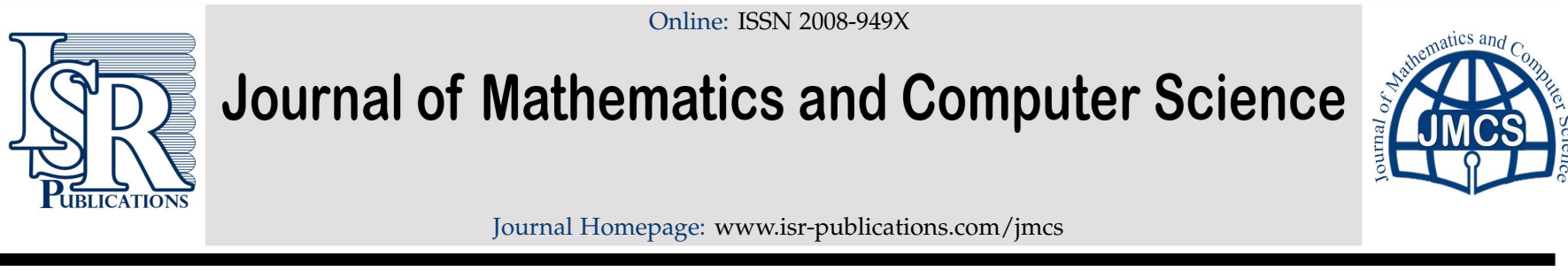

\title{
Exponential B-spline collocation method for solving the gen- eralized Newell-Whitehead-Segel equation
}

\author{
Imtiaz Wasim ${ }^{a}$, Muhammad Abbas ${ }^{a, *}$, Muhammad Kashif Iqbal ${ }^{b}$, Afzaal Mubashir Hayat $^{c}$
}

${ }^{a}$ Department of Mathematics, University of Sargodha, Sargodha, 40100, Pakistan.

${ }^{b}$ Department of Mathematics, Government College University, Faisalabad, 38000, Pakistan.

${ }^{c}$ Department of Mathematics, National College of Business Administration \& Economics, Lahore, 54660, Pakistan.

\begin{abstract}
In this work, we present a collocation method based on exponential basis spline functions for solving generalized NewellWhitehead-Segel equation. The time derivative is discretized by finite difference scheme and the exponential basis spline functions are employed to interpolate spatial derivatives. The convergence and stability of the proposed algorithm are established. Numerical results demonstrate the accuracy of the proposed method.
\end{abstract}

Keywords: Non-linear generalized Newell-Whitehead-Segel equation, exponential B-spline collocation method, convergence, stability.

2010 MSC: 65M70, 65Z05, 65N12, 65D05, 65D07.

(C)2020 All rights reserved.

\section{Introduction}

The generalized Newell-Whitehead-Segel (gNWS) equation is extensively used in fluid mechanics and its mathematical description can be stated as follows:

$$
u_{t}=m u_{x x}+a u+\psi\left(x, t, u, u_{x}, b u^{q}\right), \quad 0 \leqslant x \leqslant 1, \quad 0 \leqslant t \leqslant T,
$$

where $u$ is a function of $x$ and $t$ and $m, a, b$, are real constants with $m>0$ and $q \in Z^{+}$. The initial and the boundary conditions are represented in equations (1.2), (1.3), and (1.4), respectively.

$$
\begin{aligned}
u(x, 0) & =f(x), \\
u(0, t) & =g_{1}(t), u(1, t)=g_{2}(t), \\
u_{x}(0, t) & =h_{1}(t), u_{x}(1, t)=h_{2}(t),
\end{aligned}
$$

where $f, g_{i}$ 's, $h_{i}$ 's are known functions. By substituting $a=-1, b=1=m, q=2, \psi=b u^{q}$ into gNWS equation (1.1), we obtain Fisher's equation [3] while the values $a=1=m, b=-1, q=2, \psi=b u^{q}+u_{x}$ convert gNWS equation into Burgers-Fisher equation. Huxley equation [3] can be achieved from gNWS

\footnotetext{
*Corresponding author

Email address: muhammad.abbas@uos.edu.pk (Muhammad Abbas)

doi: $10.22436 /$ jmcs.020.04.05

Received: 2019-10-16 Revised: 2019-12-30 Accepted: 2020-01-16
} 
equation by setting $m=1, a=-\beta, b=1+\beta, q=2, \psi=b u^{q}-u^{3}$ and gNWS eqation takes the form of Burger Huxley equation when $m=1, a=-\beta, b=1+\beta, q=2, \psi=b u^{q}+u u_{x}-u^{3}$ are taken. The Newell-Whitehead-Segel (NWS) equation [14] is the special case of gNWS Eq (1.1) when $\psi=-b u^{2}$. The gNWS equation becomes Allen-Cahn [18] equation when $b=-a=4, m=1, q=3, \psi=b u^{q}$ are substituted in it while gNWS equation takes the form of Nagumo reaction diffusion equation when $\mathrm{m}=1, \mathrm{a}=\alpha, \mathrm{b}=-1, \mathrm{q}=2, \psi=b \mathrm{u}^{\mathrm{q}}+\mathrm{u}^{3}-\mathrm{u}^{2}$.

The NWS equation explains the dynamic attitude of dual blend fluid nearby bifurcation point of the Rayleigh-Benard convection of a binary fluid mixture. The layer of fluid heated from below advances a systematic design of convection cells named as Benard cells which takes place in a horizontal plane. A spontaneous change in severely heated fluid occurs which leads the hot fluid upwards while cold downwards. Because of analytical as well as experimental approachability, the phenomenon of this convection is extensively studied. The judiciously inspected examples of the self-organized nonlinear systems are the mentioned convection patterns [7]. These cells are produced due to gravity and Buoyancy forces. The primary movement is the upwelling of the warmer liquid from the heated bottom layer [16]. Two types of these shapes or patterns may be analyzed. Firstly, the liquid streamline from cylinders which might be bent or the produced spirals named as roll (stripe) pattern. Secondly, the fluid movement is separated into honey comb cells known as hexagonal shape. A part of liquid moves downwards, upwards in the middle of each cell and on the boundary between the cells, respectively while the remaining travels oppositely. For entirely different physical phenomena, the production of similar patterns can be observed, e.g., the systems with propagation of laser beams [8] in a nonlinear medium with reacting and diffusing species produce hexagonal pattern while visual cortex, human fingerprints and zebra's skin follow stripe patterns. The Rayleigh-Benard convection phenomenon and the convection cells in a gravity field are depicted in Figure 1.

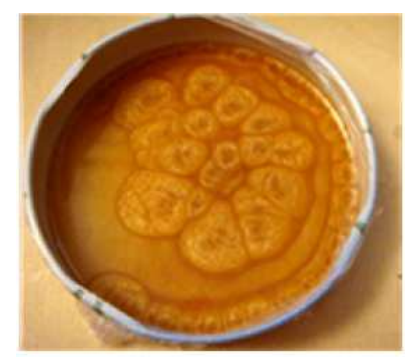

(a) Benard cells.

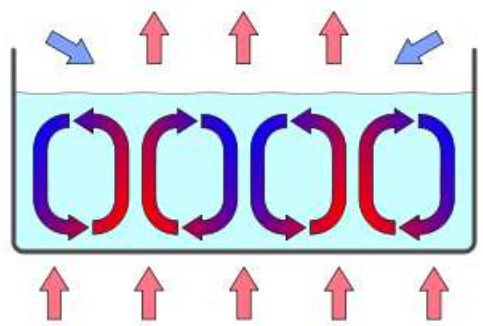

(b) Convection cells in gravity field.

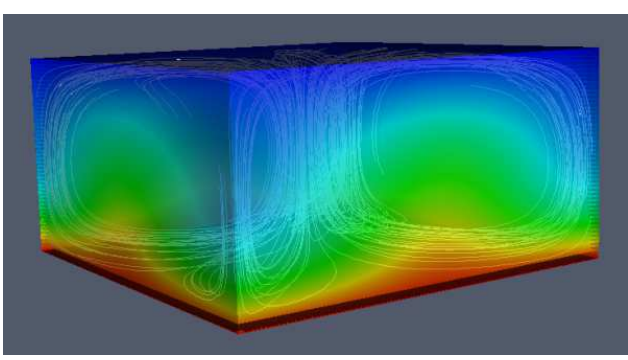

(c) Simulation of Rayleigh Benard convection in 3D.

Figure 1: Benard cells phenominon and convection cells in gravity field.

The NWS equation has been studied extensively in the last decades. Kheiri et al. [11] developed Homotopy analysis and Homotopy Pade methods for solving the modified Burgers-Korteweg-de Vries and the Newell-Whitehead equations. Ezzati and Shakibi [6] used the Adomian's Decomposition and multi-quadric qausi-interpolation techniques to obtain the solution of NWS equation. Macias-Diaz and Ramirez [12] computed numerical results for gNWS equation using finite difference algorithm. Aasaraai [1] used differential transformation method (DTM) with variable and constant coefficients to investigate the analytical solution of NWS equation. Nourazar et al. [13] developed Homotopy perturbation approach to investigate the exact solution of NWS equation. Recently, Zahra et al. [21] applied a collocation scheme based on cubic B-spline functions for the approximate solution of NWS equation using initial and boundary conditions.

Several numerical methods have been proposed for solving boundary value problems such as finite element, finite difference, spline interpolation, etc.. The B-spline method is one of the most efficient numerical methods due to its simplicity. Here we aim to use the exponential B-spline method (ExBSM) as it is capable to approximate the unknown function up to a certain smoothness. It has the potential to provide the approximation at non any point in the spatial domain with reasonable accuracy as compared to 
the typical finite difference approach. Moreover, it does not involve operators, procedure of linearization.

The main purpose of current study is to obtain the numerical solution of the proposed one dimensional nonlinear generalized Newell-Whitehead-Segel equation via exponential B-spline method. The time derivative is discretized by finite difference scheme while exponential B-spline functions interpolate spatial derivatives. The convergence and stability of the proposed method is established. The obtained numerical outcomes are show an excellent agreement with the true solutions. The computational results are compared with some other methods on the topic and it is observed that our proposed scheme preforms better in terms of accuracy and efficiency.

\section{Exponential basis functions and temporal discretization}

The time $[0, T]$ and spatial interval $[0,1]$ are partitioned into equally divided intervals and taken as pairs $\left(x_{r}, t_{k}\right)$. Here $x_{r}=a+r h, t_{k}=k \Delta t$ for $r=0,1, \ldots, N, k=0,1, \ldots, k$ where $h$ and $\Delta t$ represent spatial and time steps respectively. The exponential B-spline basis function (ExBSBF) can be stated as:

$$
\eta_{j}(x)= \begin{cases}\beta_{2}\left(\left(x_{r-2}-x\right)-\frac{1}{\sigma}\left(\sinh \left(\sigma\left(x_{r-2}-x\right)\right)\right)\right), & x \in\left[x_{r-2}, x_{r-1}\right], \\ \alpha_{1}+\beta_{1}\left(x_{r}-x\right)+\gamma e^{\sigma\left(x_{r}-x\right)}+\delta e^{-\sigma\left(x_{r}-x\right),} & x \in\left[x_{r-1}, x_{r}\right], \\ \alpha_{1}+\beta_{1}\left(x-x_{r}\right)+\gamma e^{\sigma\left(x-x_{r}\right)}+\delta e^{-\sigma\left(x-x_{r}\right)}, & x \in\left[x_{r}, x_{r+1}\right], \\ \beta_{2}\left(\left(x-x_{r-2}\right)-\frac{1}{\sigma}\left(\sinh \left(\sigma\left(x-x_{r-2}\right)\right)\right),\right. & x \in\left[x_{r+1}, x_{r+2}\right], \\ 0, & \text { else, }\end{cases}
$$

where, $\quad \alpha_{1}=\frac{\sigma h c}{\sigma h c-s}, \beta_{1}=\frac{\sigma\left(c(c-1)+s^{2}\right)}{2(\sigma h c-s)(1-c)}, \beta_{2}=\frac{\sigma}{2(\sigma h c-s)}, \gamma=\frac{e^{-\sigma h}(1-c)+s\left(e^{-\sigma h}-1\right)}{4(\sigma h c-s)(1-c)}, \delta=\frac{(c-1) e^{\sigma h}+s\left(e^{\sigma h}-1\right)}{4(\sigma h c-s)(1-c)}$, with $c=\cosh (\sigma h), s=\sinh (\sigma h)$ and $\sigma>0$ is a free parameter.

\section{Description of exponential B-spline collocation approach}

If $U(x, t)$ represents the true solution and $u(x, t)$ is the approximate solution, then using exponential B-spline functions we let $[10,19,20]$

$$
u_{j}^{k}(x, t)=\sum_{j=-1}^{N+1} \delta_{j}^{k}(t) \eta_{j}(x)
$$

where $\delta_{j}^{k}$ are to be determined. Using equations (2.1) and (3.1), we can evaluate the values of $u$ and its first two derivatives at $x_{j}$ as follows:

$$
\left\{\begin{array}{l}
u_{j}^{k}=l_{1} \delta_{j-1}^{k}+l_{2} \delta_{j}^{k}+l_{1} \delta_{j+1}^{k} \\
\left(u_{x}\right)_{j}^{k}=l_{3} \delta_{j+1}^{k}+0 \delta_{j}^{k}+\left(-l_{3}\right) \delta_{j-1}^{k} \\
\left(u_{x x}\right)_{j}^{k}=l_{4} \delta_{j-1}^{k}+\left(-2 l_{4}\right) \delta_{j}^{k}+l_{4} \delta_{j+1}^{k}
\end{array}\right.
$$

where, $l_{1}=\frac{s-\sigma h}{2(\sigma h c-s)}, l_{2}=1, l_{3}=\frac{\sigma(1-c)}{2(\sigma h c-s)}, l_{4}=\frac{s \sigma^{2}}{2(\sigma h c-s)}$. Using Eqs (1.3), (3.1), and (3.2), we can write

$$
\begin{gathered}
u\left(x_{0}, t_{k+1}\right)=l_{1} \delta_{-1}^{k+1}(t)+l_{2} \delta_{0}^{k+1}(t)+l_{1} \delta_{1}^{k+1}(t)=g_{1}\left(t_{k+1}\right), \\
u\left(x_{N}, t_{k+1}\right)=l_{1} \delta_{N-1}^{k+1}(t)+l_{2} \delta_{N}^{k+1}(t)+l_{1} \delta_{N+1}^{k+1}(t)=g_{2}\left(t_{k+1}\right) .
\end{gathered}
$$

Now implementing finite difference scheme to equation (1.1), the following expression can be obtained

$$
\frac{u_{j}^{k+1}-u_{j}^{k}}{\Delta t}=\frac{m\left(u_{x x}\right)_{j}^{k+1}+\left(m u_{x x}\right)_{j}^{k}}{2}+\frac{a(u)_{j}^{k+1}+(a u)_{j}^{k}}{2}+\frac{(\psi)_{j}^{k+1}+(\psi)_{j}^{k}}{2} .
$$


Separating terms of $k^{\text {th }}$ and $(k+1)^{\text {th }}$ time levels, equation (3.5) takes the form

$$
d_{1} u_{j}^{k+1}-d_{2}\left(u_{x x}\right)_{j}^{k+1}-\Delta t(\psi)_{j}^{k+1}=r\left(x_{j}\right),
$$

where, $d_{1}=2-a \Delta t, d_{2}=m \Delta t, d_{3}=2+a \Delta t, r\left(x_{j}\right)=d_{3} u_{j}^{k}+d_{2}\left(u_{x x}\right)_{j}^{k}+\Delta t(\psi)_{j}^{k}$. Using equation (3.2) in equation (3.6) and some simplification yields

$$
p_{1} \delta_{j-1}^{k+1}+p_{2} \delta_{j}^{k+1}+p_{1} \delta_{j+1}^{k+1}-\Delta t(\psi(u))_{j}^{k+1}=r\left(x_{j}\right), \quad j=0,1, \ldots, N,
$$

where, $p_{1}=d_{1} l_{1}-d_{2} l_{4}, p_{2}=d_{1} l_{2}+2 d_{2} l_{4}$. Eliminate the unknowns $\delta_{-1}^{k+1}$ and $\delta_{N+1}^{k+1}$ with the help of equations (3.3), (3.4), and (3.7), a system of order $(N+1) \times(N+1)$ can be generated as follows:

$$
A \delta_{j}^{k+1}-\Delta t B=C,
$$

where

$$
A=\left(\begin{array}{ccccccc}
p_{1}^{*} & 0 & 0 & & & & \\
p_{1} & p_{2} & p_{1} & & & 0 & \\
& \ddots & \ddots & \ddots & & & \\
& & 0 & & p_{1} & p_{2} & p_{1} \\
& & & & 0 & 0 & p_{1}^{*}
\end{array}\right), \delta_{j}^{k+1}=\left[\begin{array}{c}
\delta_{0}^{k+1} \\
\delta_{1}^{k+1} \\
\vdots \\
\delta_{N}^{k+1}
\end{array}\right], B=\left[\begin{array}{c}
\psi_{0}^{k+1} \\
\psi_{1}^{k+1} \\
\vdots \\
\psi_{N-1}^{k+1} \\
\psi_{N}^{k+1}
\end{array}\right], C=\left[\begin{array}{c}
r_{0}^{* k} \\
r_{1}^{k} \\
r_{2}^{k} \\
\vdots \\
r_{N-1}^{k} \\
r_{N}^{* k}
\end{array}\right]
$$

where, $r_{0}^{*}=p_{1} g_{1}(t)-l_{1} r_{0}, r_{N}^{*}=p_{1} g_{2}(t)-l_{1} r_{N}, p_{1}^{*}=l_{2} p_{1}-l_{1} p_{2}$. The above recurrence relation is solved after obtaining initial and first order approximation separately. The initial vector $u^{0}$ is computed from the initial condition (1.2). For the next approximation $u^{1}$, we use Taylor series expansion at $t=t_{0}+\Delta t$ as follows:

$$
u^{1}=u^{0}+\Delta t u_{t}^{0}+\frac{(\Delta t)^{2}}{2 !} u_{t t}^{0}+O(\Delta t)^{3}
$$

where, $u_{t}^{0}=\left(m u_{x x}+a u+\psi\right)^{0}, u_{t t}^{0}=\left(m u_{x x t}+a u_{t}+(\psi)_{t}\right)^{0}$, and the values of $u^{0}$ and its derivatives are computed by initial condition. By putting these values in equation (3.9), we achieve first order approximation as below:

$$
\mathrm{u}^{1}=\mathrm{u}^{0}+\Delta \mathrm{t}\left[\mathrm{mu} \mathrm{u}_{x x}+\mathrm{au}+\psi\right]^{0}+\frac{(\Delta \mathrm{t})^{2}}{2 !}\left[m u_{x x t}+a u_{t}+(\psi)_{t}\right]^{0}+\mathrm{O}(\Delta t)^{3} .
$$

Theorem 3.1. The rate of convergence of the presented scheme to discretize equation (1.1) is one in time direction.

Proof. Suppose $u^{k}$ be the spline approximation for exact solution $u^{k}$ at time $t=t_{k}$ and local truncation error of equation (3.6) is $e_{k}=u^{k}-u^{k}$, we have [4]

$$
e_{n+1} \leqslant \mu_{k}(\Delta t)^{2}, \quad k \geqslant 2 .
$$

By utilizing equation (3.10) for $k=1$, we obtain

$$
e_{1} \leqslant \mu_{1}(\Delta t)^{3} .
$$

Choosing $\mu=\max \left\{\mu_{1}, \mu_{2}, \ldots, \mu_{n}\right\}$ and taking global error $E_{n+1}=\sum_{k=1}^{n} e_{k}$ at $(n+1)^{\text {th }}$ time level we may obtain the following expression:

$$
\left|E_{n+1}\right|=\left|\sum_{k=1}^{n} e_{k}\right| \leqslant \sum_{k=1}^{n}\left|e_{k}\right| \leqslant \mu_{1}(\Delta t)^{3}+\sum_{k=2}^{n} \mu_{k}(\Delta t)^{2} \leqslant n \mu(\Delta t)^{2} \leqslant n \mu(T / n) \Delta t=C \Delta t,
$$

where $\Delta t \leqslant(T / n)$ and $C=\mu T$ which implies first order convergence in time direction. 


\section{Convergence of the method}

Suppose $u_{j}^{k}(x, t)=\sum_{j=-1}^{N+1} \delta_{j}(t) \eta_{j}(x)$ be the exponential B-spline approximation to the exact solution $U(x, t)$. Due to computational round off error let $S^{*}(x)=\sum_{j=-1}^{N+1} \delta_{j}^{*}(t) \eta_{j}(x)$ be the computed spline approximation to $u(x)$. Therefore, we must estimate the errors $\left\|u(x)-S^{*}(x)\right\|_{\infty}$ and $\left\|u(x)-S^{*}(x)\right\|_{\infty}$ separately to estimate the error $\|\mathfrak{u}(x)-u(x)\|_{\infty}$. Putting $S^{*}(x)$ into equation (3.8), we obtain

$$
A \delta^{*}-\Delta \mathrm{BB}^{*}=\mathrm{C}^{*} \text {. }
$$

Subtracting equation (3.10) and equation (4.1), we have

$$
A\left(\delta^{*}-\delta\right)-\Delta t\left(B^{*}-B\right)=C^{*}-C .
$$

Theorem 4.1. Suppose $\mathrm{f}(\mathrm{x}) \in \mathrm{C}^{4}[\mathrm{a}, \mathrm{b}]$ and $\left|\mathrm{f}^{4}(\mathrm{x})\right| \leqslant \mathrm{k}, \forall \mathrm{x}$ with $\mathrm{h}$ being step size of equally space partition of $[a, b]$. If $S(x)$ is the unique spline function that interpolates $f(x)$ at the nodes then $\exists$ a constant $\lambda_{j}$, s.t.,

$$
\left\|f^{j}-S^{j}\right\|_{\infty} \leqslant \lambda_{j} k h^{4-j}, j=0,1,2,3 .
$$

Proof. For proof see $[5,9]$.

Using triangular inequality and Theorem 4.1, equation (3.7) takes the following form $\left|r^{*}\left(x_{j}\right)-r\left(x_{j}\right)\right|$

$$
\begin{aligned}
& =\left|\left(d_{1} S^{*}\left(x_{j}\right)-d_{2} S_{x x}^{*}\left(x_{j}\right)-\Delta t \psi\left(S^{*}\left(x_{j}\right)\right)\right)-\left(d_{1} u_{j}^{k+1}-d_{2}\left(u_{x x}\right)_{j}^{k+1}-\Delta t(\psi)_{j}^{k+1}\right)\right| \\
& \leqslant\left|d_{1}\right|\left|S^{*}\left(x_{j}\right)-U\left(x_{j}\right)\right|+d_{2}\left|S_{x x}^{*}\left(x_{j}\right)-u_{x x}\left(x_{j}\right)\right|+\Delta t\left|\psi\left(x_{j}, S^{*}\left(x_{j}\right)\right)-\psi\left(x_{j}, U\left(x_{j}\right)\right)\right| \\
& \leqslant d_{1} \kappa \lambda_{0} h^{4}+d_{2} \kappa \lambda_{2} h^{2}+\beta\left(\left|S^{*}\left(x_{j}\right)-U\left(x_{j}\right)\right|\right),
\end{aligned}
$$

where, $\left\|\psi^{\prime}(z)\right\| \leqslant \beta, z \in R^{3}$ [15]. Finally, we are able to write

$$
\left\|C^{*}-C\right\| \leqslant d_{1} \kappa \lambda_{0} h^{4}+d_{2} \kappa \lambda_{2} h^{2}+\beta\left(\left|S^{*}\left(x_{j}\right)-U\left(x_{j}\right)\right|\right) .
$$

Again using Theorem 4.1 yields

$$
\left\|C^{*}-C\right\| \leqslant d_{1} \kappa \lambda_{0} h^{4}+d_{2} \kappa \lambda_{2} h^{2}+\beta \kappa \lambda_{0} h^{4} .
$$

Also we can write

$$
\left\|C^{*}-C\right\| \leqslant M_{1} h^{2}
$$

where $M_{1}=d_{1} \kappa \lambda_{0} h^{2}+d_{2} \kappa \lambda_{2}+\beta \kappa \lambda_{0} h^{2}$.

Now using Jacobian for nonlinear term on L.H.S. of equation (4.2), we obtain the following equation

$$
\left\|B^{*}-B\right\|=\left(\frac{\partial \varphi\left(\xi_{1}\right)}{\partial u} J\left(\delta^{*}-\delta\right)\right),
$$

where $\xi_{1} \in(0,1)$ and $J$ is Jacobian given as

$$
J=\left(\begin{array}{ccccccc}
0 & 0 & 0 & & & & \\
l_{1} & l_{2} & l_{1} & & & 0 & \\
& \ddots & \ddots & \ddots & & & \\
& & & & l_{1} & l_{2} & l_{1} \\
& & 0 & & 0 & 0 & 0
\end{array}\right) .
$$

Substituting equation (4.4) into equation (4.2), the following expression is obtained

$$
W\left(\delta^{*}-\delta\right)=\left(C^{*}-C\right)
$$


where $W=A+\frac{\partial \varphi\left(\xi_{1}\right)}{\partial u} J$. Since matrix $W$ is strictly diagonally dominant so non-singular, $W^{-1}$ exists, hence equation (4.5) implies

$$
\left(\delta^{*}-\delta\right)=W^{-1}\left(C^{*}-C\right) .
$$

Taking norm on both sides of the above equation and using equation (4.3), we obtain

$$
\left\|\delta^{*}-\delta\right\|_{\infty} \leqslant\left\|W^{-1}\right\| M_{1} h^{2}
$$

Suppose $\gamma_{j}$ is the sum of $j^{\text {th }}$ row of matrix $W=\left[v_{j, i}\right]$ for $i=0,1, \ldots, N$, then we have

$$
\begin{cases}\gamma_{0}=l_{2} p_{1}-l_{1} p_{2}, & \text { if } \quad j=0 \\ \gamma_{j}=2 p_{1}+p_{2}, & \text { if } \quad 1 \leqslant j \leqslant N-1, \\ \gamma_{N}=l_{2} p_{1}-l_{1} p_{2}, & \text { if } \quad j=N .\end{cases}
$$

From the properties of inverse of matrices, we can write

$$
\sum_{j=0}^{N} v_{i, j}^{-1} \gamma_{j}=1
$$

where $v_{i, j}^{-1}$ are the entries of $W^{-1}$.

$$
\left\|W^{-1}\right\|=\sum_{j=0}^{N}\left\|v_{i, j}^{-1}\right\| \leqslant \frac{1}{\min \left(\gamma_{j}\right)}=\frac{1}{v_{l}} \leqslant \frac{1}{\left|v_{l}\right|^{\prime}}
$$

where $l$ is some integer between 0 and $N$. Putting equation (4.7) in equation (4.6) implies the relation

$$
\left\|\delta^{*}-\delta\right\|_{\infty} \leqslant \beta_{2} h^{2}
$$

where $\beta_{2}=\frac{M_{1}}{v_{l}}$.

Lemma 4.2. The exponential B-Spline basis functions satisfy

$$
\left|\sum_{j=-1}^{N} \eta_{j}(x)\right| \leqslant 3, \quad 0 \leqslant x \leqslant 1
$$

Proof. We know that

$$
\left|\sum_{j=-1}^{N+1} \eta_{j}(x)\right| \leqslant \sum_{j=-1}^{N+1}\left|\eta_{j}(x)\right|
$$

At any knot $x_{j}$ we have

$$
\sum_{j=-1}^{N+1}\left|\eta_{j}(x)\right|=\left|\eta_{j-1}(x)\right|+\left|\eta_{j}(x)\right|+\left|\eta_{j+1}(x)\right|=\left|l_{1}\right|+\left|l_{2}\right|+\left|l_{1}\right| \leqslant 2 .
$$

Also in each subinterval $x_{j-1} \leqslant x \leqslant x_{j}$

$$
\eta_{j}\left(x_{j}\right)=l_{2}, \eta_{j-1}\left(x_{j-1}\right)=l_{2}, \eta_{j+1}\left(x_{j}\right)=l_{1}, \eta_{j-2}\left(x_{j-1}\right)=l_{1} .
$$

Hence for any $x_{j-1} \leqslant x \leqslant x_{j}$, it is verified that

$$
\sum_{j=-1}^{N+1}\left|\eta_{j}(x)\right|=\left|\eta_{j-2}(x)\right|+\left|\eta_{j-1}(x)\right|+\left|\eta_{j}(x)\right|+\left|\eta_{j+1}(x)\right| \leqslant 3,
$$

which completes the proof. 
Since

$$
S^{*}(x)-u(x)=\sum_{j=-1}^{N+1}\left(\delta_{j}^{*}-\delta_{j}\right) \eta_{j}(x) .
$$

Applying norm on both sides of the above equation and using equations (4.8) and (4.9) enables us to write

$$
\left\|S^{*}(x)-u(x)\right\|=\left\|\sum_{j=-1}^{N+1}\left(\delta_{j}^{*}-\delta_{j}\right) \eta_{j}(x)\right\|=\left|\sum_{j=0}^{N} \eta_{j}(x)\right|\left\|\delta_{j}^{*}-\delta_{j}\right\| \leqslant 3 \beta_{2} h^{2} .
$$

Theorem 4.3. If $\mathrm{u}(\mathrm{x})$ is the exponential B-spline collocation approximation to exact solution $\mathrm{U}(\mathrm{x})$ then the method has 2 nd order convergence

$$
\|\mathrm{u}(\mathrm{x})-\mathrm{u}(\mathrm{x})\| \leqslant \epsilon \mathrm{h}^{2},
$$

where $\epsilon=\lambda_{0} \mathrm{Kh}^{2}+3 \beta_{2}$ is a finite constant.

Proof. Using triangular inequality and Theorem 4.1, the following relation may be achieved

$$
\begin{aligned}
\|\mathfrak{u}(x)-\mathrm{u}(x)\| & =\left\|\mathfrak{u}(x)-\mathrm{S}^{*}(x)+S^{*}(x)-\mathrm{u}(x)\right\| \\
& \leqslant\left\|S^{*}(x)-\mathrm{u}(x)\right\|+\left\|\mathfrak{u}(x)-S^{*}(x)\right\| \leqslant \lambda_{0} k h^{4}+3 \beta_{2} h^{2}=\epsilon h^{2},
\end{aligned}
$$

where $\epsilon=\lambda_{0} k h^{2}+3 \beta_{2}$.

Now if $u(x, t)$ approximates the exact solution $u(x, t)$, then

$$
\|u(x, t)-u(x, t)\| \leqslant \omega\left(\Delta t+h^{2}\right),
$$

where $\omega$ is constant. Hence our method is of first order convergence in time while second order in spatial direction.

\section{Stability}

For Von Neumann stability analysis, consider $\psi=b u^{q}$ in equation (1.1), the equation (3.7) takes the following form

$$
\mathrm{q}_{1} \delta_{j-1}^{\mathrm{k}+1}+\mathrm{q}_{2} \delta_{j}^{\mathrm{k}+1}+\mathrm{q}_{1} \delta_{j+1}^{\mathrm{k}+1}=\mathrm{q}_{3} \delta_{j-1}^{\mathrm{k}}+\mathrm{q}_{4} \delta_{j}^{\mathrm{k}}+\mathrm{q}_{3} \delta_{j+1}^{\mathrm{k}}
$$

where, $d_{4}=b \Delta t, q_{1}=d_{1} l_{1}-d_{2} l_{4}-d 4\left(l_{1}\right)^{q}, q_{2}=d_{1} l_{2}+2 d_{2} l_{4}-d 4\left(l_{2}\right)^{q}, q_{3}=d_{3} l_{1}+d_{2} l_{4}+d_{4}\left(l_{1}\right)^{q}$, $\mathrm{q}_{4}=\mathrm{d}_{3} l_{2}-2 \mathrm{~d}_{2} \mathrm{l}_{4}+\mathrm{d}_{4}\left(\mathrm{l}_{2}\right)^{\mathrm{q}}$. Put $\delta_{j}^{k}=\rho^{\mathrm{k}} e^{i \eta j h}$ [17] in equation (5.1), we obtain

$$
q_{1} \rho^{k+1} e^{i \eta(j-1) h}+q_{2} \rho^{k+1} e^{i \eta(j) h}+q_{1} \rho^{k+1} e^{i \eta(j+1) h}=q_{3} \rho^{k} e^{i \eta(j-1) h}+q_{4} \rho^{k} e^{i \eta(j) h}+q_{3} \rho^{k} e^{i \eta(j+1) h} .
$$

Take $\phi=\eta \mathrm{h}$ and dividing both sides by $\rho^{k} e^{i \eta(j) h}$ yields

$$
\rho\left(q_{1} e^{-i \phi}+q_{2}+q_{1} e^{i \phi}\right)=q_{3} e^{-i \phi}+q_{4}+q_{3} e^{i \phi} .
$$

Since $e^{i \phi}=\cos (\phi)+i \sin (\phi)$, so above equation yields

$$
\rho=\frac{2 q_{3} \cos (\phi)+p_{4}}{2 q_{1} \cos (\phi)+q_{2}} .
$$

Substituting equations (3.2), (3.6), and (5.1) into equation (5.2) and after simplification the following relation can be obtained as

$$
\rho=-1+\frac{2^{2+q}(\mu \cos (\phi)-v)}{2 b v \Delta t\left(\frac{\mu}{v}\right)^{q} \cos (\phi)+2^{q}\left(v(-2+(a+b) \Delta t)-s v_{1}+\left(v_{2}+s\left(-2+a \Delta t+v_{1}\right)\right) \cos (\phi)\right)},
$$

where $v=\sigma h c-s, v_{1}=m \Delta t \sigma^{2}, \mu=s-h \sigma, v_{2}=h(2-a \Delta t) \sigma, c=\cosh (h \sigma), s=\sin (h \sigma)$. It is obvious from Eq. (5.3) that $|\rho|<1$ which demonstrates the proposed method unconditionally stable. 


\section{Numerical experiments}

The numerical method presented in this paper is tested for getting solution of the generalized NewellWhitehead-Segel equation for the numerical accuracy. Throughout the paper, we set free parameter $\sigma=1.175$. All the computations are performed in MATLAB R2015b. In the first three examples, the initial and Dirichlet boundary conditions are obtained from the exact solution while in the last example Neumann boundary conditions are used. The following formulas are used to compute the numerical results.

$$
\text { Absolute error }=\left|u_{j}-u_{j}^{k}\right|, \quad L_{\infty}=\max _{j}\left|u_{j}-u_{j}\right| .
$$

Example 6.1. Consider gNWS equation (1.1) with $a=2, m=1, q=2, b=-3, \psi=b u^{q}$ for which the exact solution given in $[1,21]$ is

$$
u(x, t)=\frac{-2 \lambda e^{2 t}}{-2+3 \lambda\left(1-e^{2 t}\right)}
$$

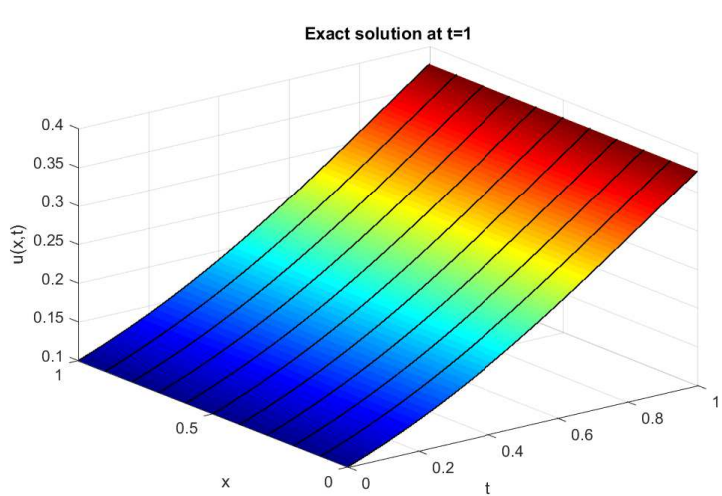

(a) Exact

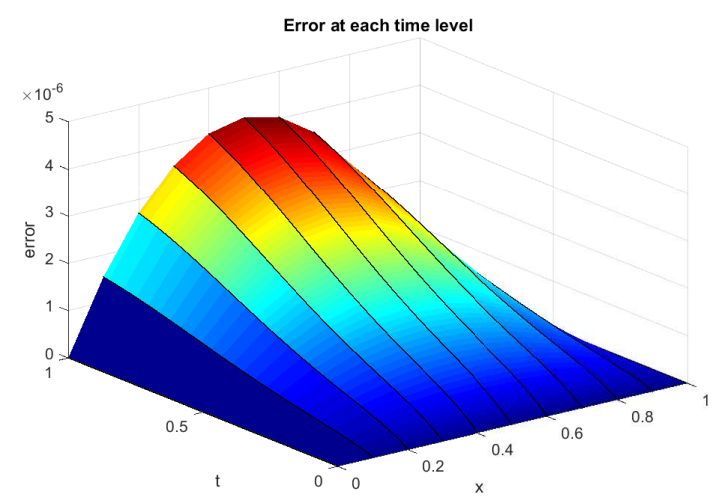

(c) $3 \mathrm{D}$ error

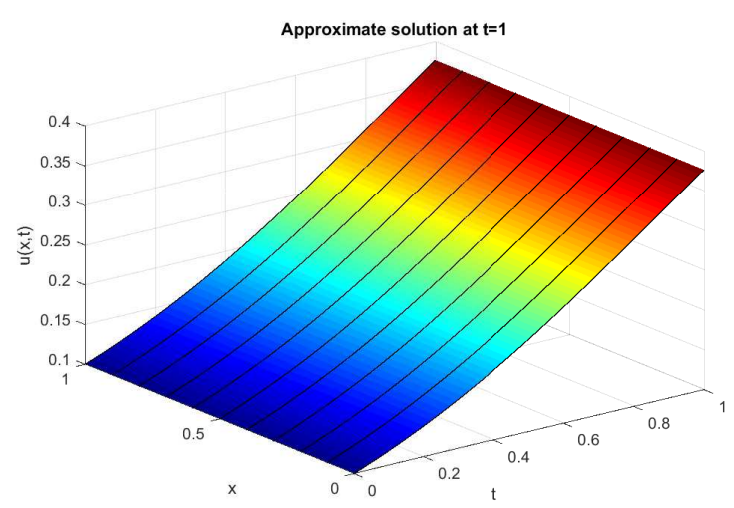

(b) Approximate

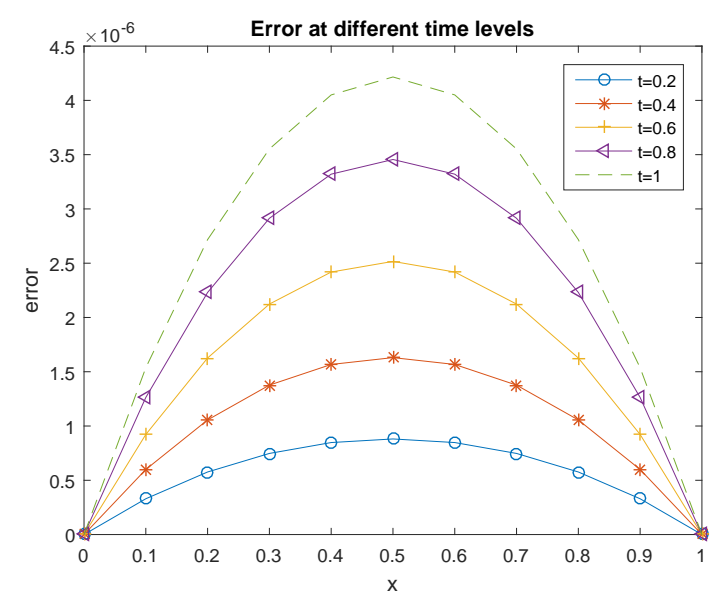

(d) $2 \mathrm{D}$ error

Figure 2: Solutions and Error graphs for $t \in[0,1]$ of Example 6.1.

The absolute maximum errors of Example 6.1 are provided in Table 1 at different time levels and knots. The numerical results of ExBSM are compared with the existing methods named as Uniform cubic B-spline (UCBS) [21], Trigonometric cubic B-spline (TCBS) [21], Extended cubic B-spline (ECBS) [21]. Figure 2 exhibits the solutions and errors graphically. It is obvious that the proposed method is more reliable and efficient as compared to others. 
Table 1: Absolute maximum errors at $\lambda=0.1$ of Example 6.1.

\begin{tabular}{|c|c|c|c|c|c|}
\hline $\mathrm{x}$ & $\mathrm{t}$ & ExBSM & UCBS[21] & TCBS[21] & ECBS[21] \\
\hline 0.2 & 0.2 & $5.760 \mathrm{E}-07$ & $8.323 \mathrm{E}-04$ & $8.295 \mathrm{E}-04$ & $6.068 \mathrm{E}-04$ \\
\hline 0.6 & & $8.471 \mathrm{E}-07$ & $1.226 \mathrm{E}-03$ & $1.222 \mathrm{E}-03$ & $9.013 \mathrm{E}-04$ \\
\hline 0.8 & & $5.760 \mathrm{E}-07$ & $8.323 \mathrm{E}-04$ & $8.295 \mathrm{E}-04$ & $6.068 \mathrm{E}-04$ \\
\hline 0.2 & 1.0 & $2.714 \mathrm{E}-06$ & $1.991 \mathrm{E}-05$ & $3.239 \mathrm{E}-05$ & $6.339 \mathrm{E}-05$ \\
\hline 0.6 & & $4.046 \mathrm{E}-06$ & $4.932 \mathrm{E}-06$ & $2.342 \mathrm{E}-05$ & $8.366 \mathrm{E}-05$ \\
\hline 0.8 & & $2.714 \mathrm{E}-06$ & $1.991 \mathrm{E}-05$ & $3.239 \mathrm{E}-05$ & $6.339 \mathrm{E}-05$ \\
\hline
\end{tabular}

Example 6.2. Consider gNWS equation (1.1) with $a=1=m, q=4, b=-1, \psi=b u^{q}$ and the exact solution given in $[13,21]$ is

$$
u(x, t)=\left(\frac{1}{2} \tanh \left(\frac{-2}{2 \sqrt{10}}\left(x-\frac{7 t}{\sqrt{10}}\right)\right)+\frac{1}{2}\right)^{\frac{-2}{3}}
$$

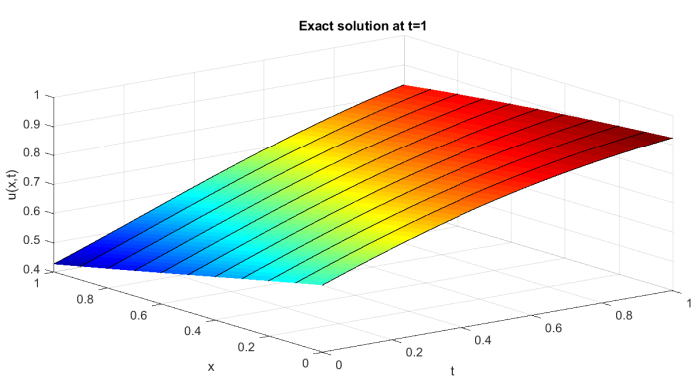

(a) Exact

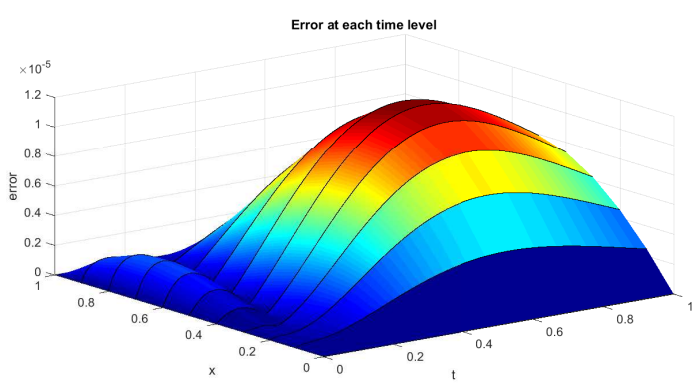

(c) $3 \mathrm{D}$ error

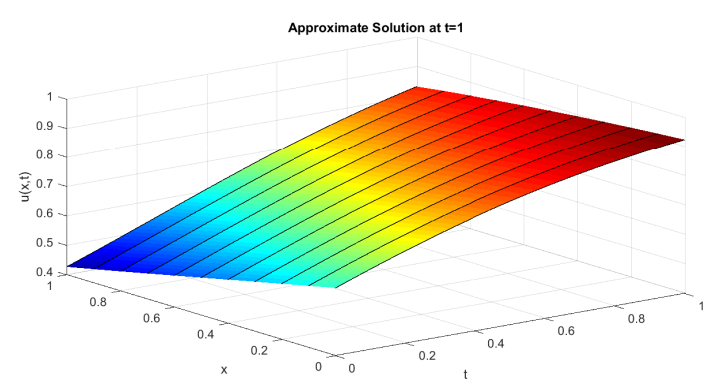

(b) Approximate

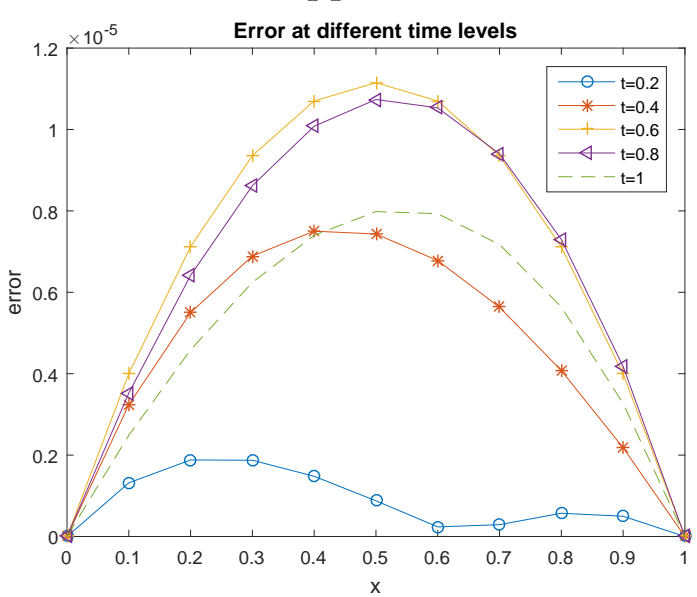

(d) $2 \mathrm{D}$ error

Figure 3: Solutions and Error graphs for $t \in[0,1]$ of Example 6.2.

The error terms of Example 6.2 at different time levels and knots are provided in Table 2. The numerical outputs of ExBSM are compared with the methods (UCBS, TCBS, ECBS). The solutions and error terms are displayed in Figure 3. It can be seen that proposed scheme is more reliable and efficient.

Example 6.3. Consider gNWS equation (1.1) with $a=3, b=-4, m=1, q=3, \psi=b u^{q}$ with the true solution given in $[13,21]$ as

$$
u(x, t)=\frac{e^{x \sqrt{6}} \sqrt{\left(\frac{3}{4}\right)}}{e^{x \sqrt{6}}+e^{\left(\frac{x \sqrt{6}}{2}-\frac{9 t}{2}\right)}} .
$$


Table 2: Absolute maximum errors for $x, t \in[0,1]$ of Example 6.2.

\begin{tabular}{|l|l|l|l|l|l|}
\hline$x$ & $t$ & ExBSM & UCBS[21] & TCBS[21] & ECBS[21] \\
\hline 0.2 & 0.2 & $1.881 \mathrm{E}-06$ & $3.800 \mathrm{E}-04$ & $3.951 \mathrm{E}-04$ & $9.673 \mathrm{E}-04$ \\
\hline 0.6 & & $1.985 \mathrm{E}-06$ & $2.890 \mathrm{E}-04$ & $3.111 \mathrm{E}-04$ & $8.863 \mathrm{E}-04$ \\
\hline 0.8 & & $2.122 \mathrm{E}-06$ & $1.120 \mathrm{E}-04$ & $1.270 \mathrm{E}-04$ & $4.224 \mathrm{E}-04$ \\
\hline 0.2 & 1.0 & $7.143 \mathrm{E}-06$ & $1.080 \mathrm{E}-03$ & $1.103 \mathrm{E}-03$ & $2.251 \mathrm{E}-03$ \\
\hline 0.6 & & $1.107 \mathrm{E}-05$ & $1.703 \mathrm{E}-03$ & $1.734 \mathrm{E}-03$ & $3.558 \mathrm{E}-03$ \\
\hline 0.8 & & $7.534 \mathrm{E}-06$ & $1.172 \mathrm{E}-03$ & $1.192 \mathrm{E}-03$ & $2.441 \mathrm{E}-03$ \\
\hline
\end{tabular}

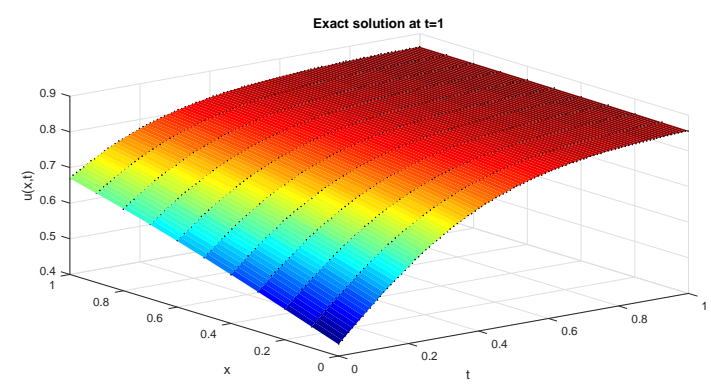

(a) Exact

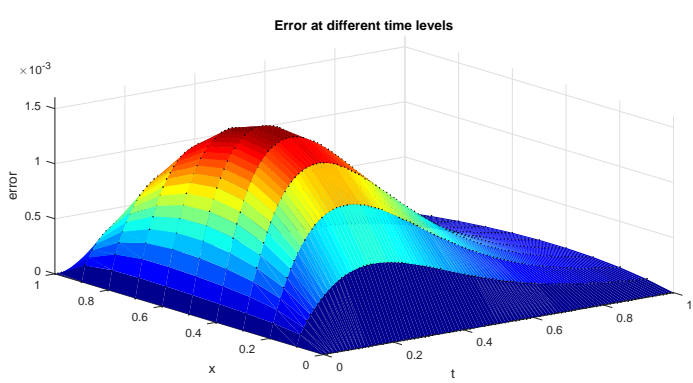

(c) $3 \mathrm{D}$ error

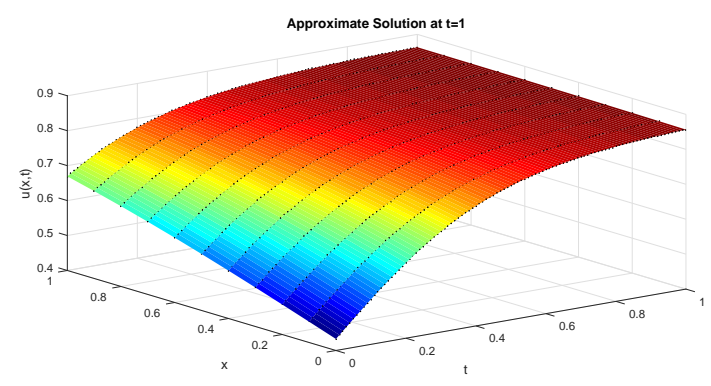

(b) Approximate

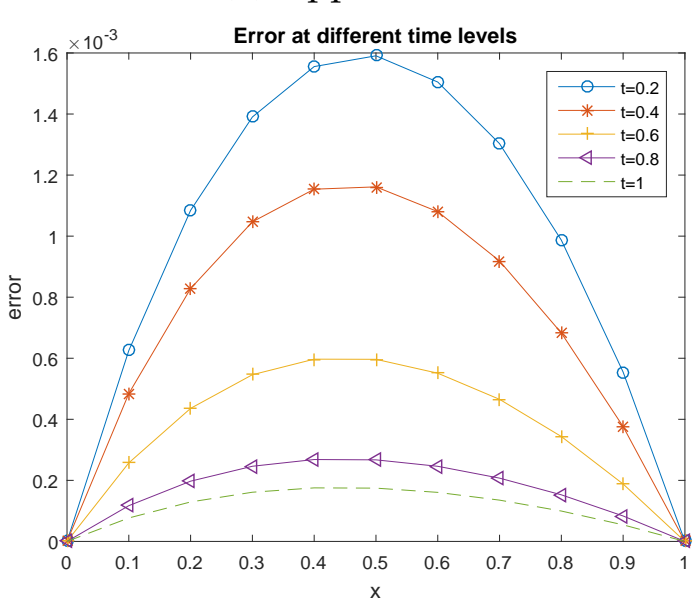

(d) $2 \mathrm{D}$ error

Figure 4: Solutions and Error graphs for $t \in[0,1]$ of Example 6.3.

Table 3: Absolute maximum errors of Example 6.3.

\begin{tabular}{|l|l|l|l|l|l|}
\hline $\mathrm{x}$ & $\mathrm{t}$ & $\mathrm{ExBSM}$ & $\mathrm{UCBS}[21]$ & $\mathrm{TCBS}[21]$ & $\mathrm{ECBS}[21]$ \\
\hline 0.2 & 0.2 & $1.100 \mathrm{E}-03$ & $6.129 \mathrm{E}-02$ & $6.134 \mathrm{E}-02$ & $5.166 \mathrm{E}-02$ \\
\hline 0.6 & & $1.500 \mathrm{E}-03$ & $8.030 \mathrm{E}-02$ & $8.036 \mathrm{E}-02$ & $6.461 \mathrm{E}-02$ \\
\hline 0.8 & & $9.883 \mathrm{E}-04$ & $4.857 \mathrm{E}-02$ & $4.862 \mathrm{E}-02$ & $3.721 \mathrm{E}-02$ \\
\hline 0.2 & 1.0 & $1.100 \mathrm{E}-03$ & $1.560 \mathrm{E}-02$ & $1.563 \mathrm{E}-02$ & $1.518 \mathrm{E}-03$ \\
\hline 0.6 & & $1.500 \mathrm{E}-03$ & $9.878 \mathrm{E}-03$ & $9.969 \mathrm{E}-03$ & $9.679 \mathrm{E}-03$ \\
\hline 0.8 & & $9.883 \mathrm{E}-04$ & $2.862 \mathrm{E}-03$ & $2.915 \mathrm{E}-03$ & $9.118 \mathrm{E}-03$ \\
\hline
\end{tabular}

For Example 6.3., the absolute maximum errors are tabulated in Table 3. A comparison of the ExBSM with the existing methods (UCBS, TCBS, ECBS) can be analyzed. Figure 4 exhibits the solutions and errors graphs. It may be observed that our method is more efficient, accurate and well organized. 
Example 6.4. Consider gNWS equation (1.1) with $a=0=b, m=1, q=3, \psi=b u^{q}+u(\alpha-u)(1-u), \alpha=$ $0.5, l=1, \eta=0.3$ with the exact solution given in $[17,21]$ as

$$
u(x, t)=\left\{\begin{aligned}
& 0.3+0.04201052049 t-0.002794271318 t^{2}-0.0001237074665 t^{3} \\
&-\left(6.498086711 \times(10)^{-10} t+2.063100590 \times(10)^{-9} t^{2}-1.502107790 \times(10)^{-9} t^{3}\right) x^{2}(l-x)^{2} \\
&-\left(5.530644662 \times(10)^{-9} t-1.727330167 \times(10)^{-8} t^{2}+1.237824484 \times(10)^{-8} t^{3}\right) x^{3}(l-x)^{3}
\end{aligned}\right.
$$

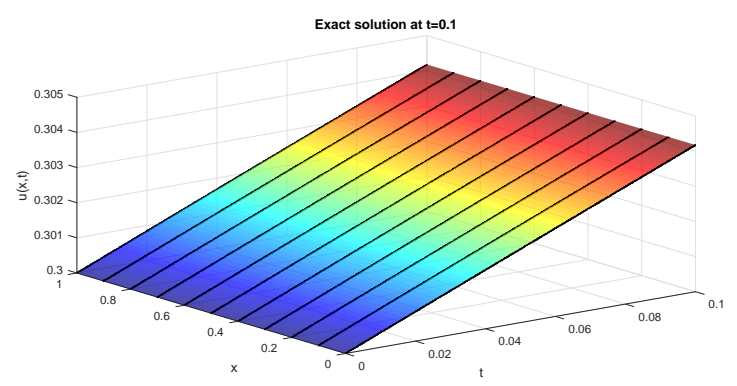

(a) Exact

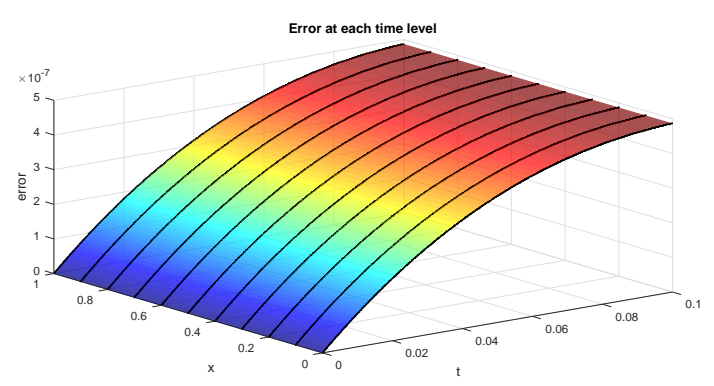

(c) 3D error

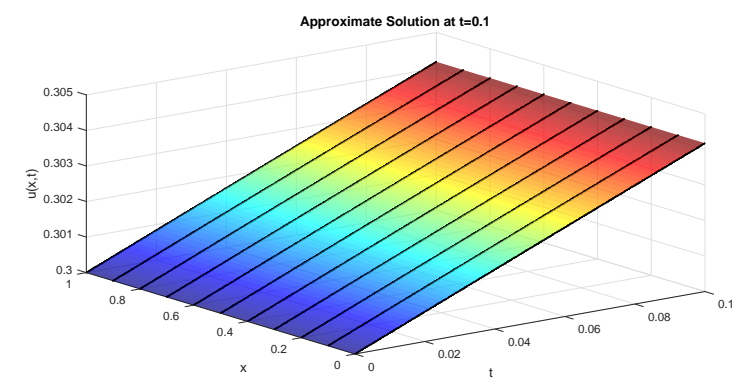

(b) Approximate

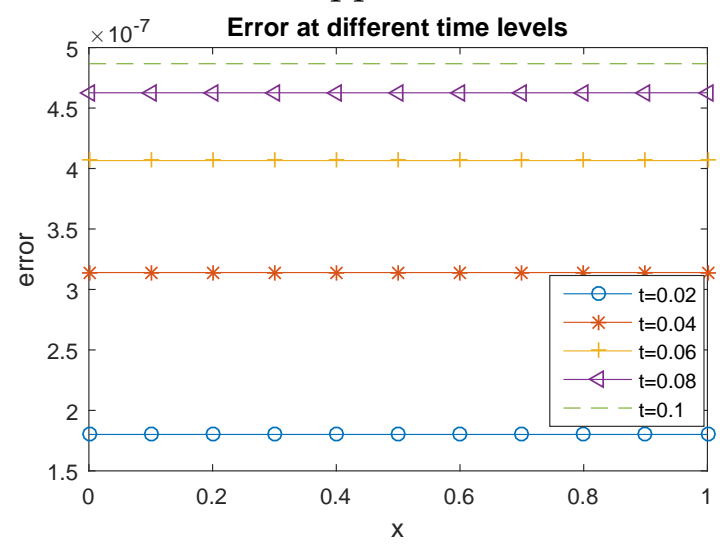

(d) $2 \mathrm{D}$ error

Figure 5: Solutions and Error graphs for $t \in[0,0.1]$ of Example 6.4.

Table 4: Exact and approximate solution of Example 6.4.

\begin{tabular}{|l|l|l|l|l|l|l|l|l|}
\hline$x$ & $t$ & ExBSM & UCBS[21] & AVF[17] & $t$ & proposed & UCBS[21] & AVF[17] \\
\hline 0.1 & 0.02 & 0.3008 & 0.2991 & 0.3026 & 0.08 & 0.3033 & 0.2966 & 0.3107 \\
\hline 0.5 & & 0.3008 & 0.2991 & 0.3026 & & 0.3033 & 0.2966 & 0.3107 \\
\hline 0.9 & & 0.3008 & 0.2991 & 0.3026 & & 0.3033 & 0.2966 & 0.3107 \\
\hline
\end{tabular}

In Table 4, the exact and approximate solutions of Example 6.4 are listed. The numerical results obtained by ExBSM are in close agreement with the true solution. It can be seen that our method is more accurate as compared to the methods named (UCBS) and Variational formulation (VF) [17]. Figure 5 depict the solutions and errors patterns.

\section{Conclusion:}

The current study presents direct implementation of exponential B-spline method to generalizedWhitehead-Segel equation to obtain its numerical solution. For this purpose, three different types of boundary conditions are considered. The convergence of the proposed method is established both in 
space and time directions. The proposed scheme has been shown to be stable without any condition. The obtained numerical results are in good agreement with the exact analytical solutions. A comparison shows that the proposed method furnishes more accurate results as compared to the methods named as Uniform cubic B-spline (UCBS) [21], Trigonometric cubic B-spline (TCBS) [21], Extended cubic B-spline (ECBS) [21], and Variational formulation (VF) [17].

\section{References}

[1] A. Aasaraai, Analytic solution for Newell-Whitehead-Segel equation by differential transform method, Middle-East J. Sci. Res., 10 (2011), 270-273. 1, 6.1

[2] M. Abbas, A. A. Majid, A. I. M. Ismail, A. Rashid, The application of cubic trigonometric B-spline to the numerical solution of the hyperbolic problems, Appl. Math. Comput., 239 (2014), 74-88.

[3] E. Babolian, J. Saeidian, Analytic approximate solutions to Burgers, Fisher, Huxley equations and two combined forms of these equations, Commun. Nonlinear Sci. Numer. Simul., 14 (2009), 1984-1992. 1

[4] C. Clavero, J. C. Jorge, F. Lisbona, Uniformly convergent scheme on a nonuniform mesh for convection-diffusion parabolic problems, J. Comput. Appl. Math., 154 (2003), 415-429. 3

[5] C. de Boor, On the convergence of odd degree spline interpolation, J. Approximation Theory, 1 (1968), 452-463. 4

[6] R. Ezzati, K. Shakibi, Using Adomian's decomposition and multiquadric quasi-interpolation methods for solving NewellWhitehead equation, Procedia Comput. Sci., 3 (2011), 1043-1048. 1

[7] A. V. Getling, Rayleigh-Bénard Convection: Structures and Dynamics, World Scientific Publishing Co., River Edge, (1998). 1

[8] A. A. Golovin, A. A. Nepomnyashchy, General Aspects of Pattern Formation, in: Self-assembly, pattern formation and growth phenomena in nano-systems, 2007 (2007), 1-54. 1

[9] C. A. Hall, On error bounds for spline interpolation, J. Approximation Theory, 1 (1968), 209-218. 4

[10] M. K. Iqbal, M. Abbas, I. Wasim, New cubic B-spline approximation for solving third order EmdenFlower type equations, Appl. Math. Comput., 331 (2018), 319-333. 3

[11] H. Kheiri, N. Alipour, R. Dehghani, Homotopy analysis and Homotopy Pad methods for the modified Burgers-Korteweg-de Vries and the Newell-Whitehead equations, Math. Sci. Q. J., 5 (2011), 33-50. 1

[12] J. E. Macias-Diaz, J. Ruiz-Ramirez, A non-standard symmetry-preserving method to compute bounded solutions of a generalized Newell-Whitehead Segel equation, Appl. Numer. Math., 61 (2011), 630-640. 1

[13] S. S. Nourazar, M. Soori, A. Nazari-Golshan, On the exact solution of Newell-Whitehead-Segel equation using the homotopy perturbation method, Aust. J. Basic Appl. Sci., 5 (2011), 1400-1411. 1, 6.2, 6.3

[14] J. Patade, S. Bhalekar, Approximate analytical solutions of Newell-Whitehead-Segel equation using a new iterative method, World J. Modell. Simul., 11 (2015), 94-103. 1

[15] W. Rudin, Principles of Mathematical analysis, McGraw-Hill Book Co., New York-Auckland-Düsseldorf, (1976). 4

[16] UC. San Diego, Rayleigh-Benard Convection, Department of Physics, (2009). 1

[17] R. A. Van Gorder, K. Vajravelu, A variational formulation of the Nagumo reaction diffusion equation and the Nagumo telegraph equation, Nonlinear Anal. Real World Appl., 11 (2010), 2957-2962. 5, 6.4, 4, 6, 7

[18] A. Voigt, Asymptotic behavior of solutions to the Allen-Cahn equation in spherically symmetric domains, Caesar-Preprints, (2001), 1-8. 1

[19] I. Wasim, M. Abbas, M. Amin, Hybrid b-spline collocation method for solving the generalized Burgers-Fisher and BurgersHuxley equations, Math. Probl. Eng., 2018 (2018), 18 pages. 3

[20] I. Wasim, M. Abbas, M. K. Iqbal, Numerical solution of modified forms of Camassa-Holm and Degasperis-Procesl equations via quartic B-spline collocation method, Commun. Math. Appl., 9 (2018), 393-409. 3

[21] W. K. Zahra, W. A. Ouf, M. S. El-Azab, Cubic B-spline collocation algorithm for the numerical solution of Newell Whitehead Segel type equations, Electron. J. Math. Anal. Appl., 2 (2014), 81-100. 1, 6.1, 6, 1, 6.2, 6.3, 2, 3, 6.4, 4, 7 